\title{
High-temperature thermoelectric characterization of filled strontium barium niobates: power factors and carrier concentrations
}

\author{
Jason H. Chan, ${ }^{\text {a) }}$ Jonathan A. Bock, Hanzheng Guo, Susan Trolier-McKinstry, and Clive A. Randall \\ Materials Science and Engineering Department and Materials Research Institute, The Pennsylvania State \\ University, University Park, PA 16802
}

(Received 25 August 2016; accepted 5 January 2017)

\begin{abstract}
Thermoelectric properties of oxygen-deficient filled strontium barium niobates (SBN, $\left.\mathrm{Sr}_{x} \mathrm{Ba}_{6-x} \mathrm{Nb}_{10} \mathrm{O}_{30-\delta}\right)$ in the composition range from the barium end member to a Sr:Ba ratio of 80:20 were investigated. The electrical conductivity, Seebeck coefficients, and power factors for ceramic samples annealed at $1300-1310{ }^{\circ} \mathrm{C}$ for $30 \mathrm{~h}$ under forming gas $\left(\sim 10^{-16} \mathrm{pO}_{2} \mathrm{~atm}\right)$ were evaluated from $\sim 350$ to $970 \mathrm{~K}$. The conduction mechanism in the filled SBNs was found to be similar to that of the heavily-reduced unfilled SBNs reported in literature. However, relative to the unfilled counterparts heat-treated at $10^{-16} \mathrm{~atm} \mathrm{pO}_{2}$, larger power factors were observed in the filled SBNs. The thermoelectric performance of these filled SBNs was composition-sensitive; lower $\mathrm{Sr}$ contents showed higher electrical conductivities, and power factors. Electron diffraction and Hall experiments suggest that both mobility and carrier concentration are enhanced with decreasing Sr. For ceramic samples, the highest power factors achievable were found for low $\mathrm{Sr}$, heavily-reduced filled compositions.
\end{abstract}

\section{INTRODUCTION}

Thermoelectric materials may play a pivotal role in improving energy sustainability. Thermoelectric devices are not expected to replace current methods of energy generation, but instead, to harness the waste heat that evolves from burning of fossil fuels in automobiles or power plants. Thermoelectric generators are especially attractive in that they are silent and house no moving components. $^{1}$ Other advantages include reliability (exceeding 100,000 h of steady-state operation) and the scalability of size to meet the required application. ${ }^{2}$

The primary factor that prevents thermoelectrics from wider implementation is the low efficiency-to-cost ratio. ${ }^{1}$ For example, tellurium, a component in some of the most-utilized thermoelectrics, has a cost that is comparable to $\mathrm{Pt}^{3}$ SiGe alloys are among the most efficient thermoelectric materials at high temperatures, but only possess an efficiency of around $8 \% .{ }^{4}$ Currently, thermoelectrics are constrained to niche uses, especially where cost concerns are outweighed by other factors, such as in military or space applications. ${ }^{2}$ Thus, there is interest in developing less-expensive alternative thermoelectrics.

Despite the ubiquity of oxides in other electronic applications, only a handful of useful oxide thermoelectrics have been reported. Layered cobaltites still lead by almost a factor of 2 in terms of $\mathrm{zT}^{5}$ However, oxygen-deficient unfilled strontium barium niobate (or $\mathrm{SBN}, \mathrm{Sr}_{5-x} \mathrm{Ba}_{x} \mathrm{Nb}_{10} \mathrm{O}_{30}$ ),

Contributing Editor: Gary L. Messing

${ }^{a}$ Address all correspondence to this author.

e-mail: Jason.chan1631@gmail.com

DOI: $10.1557 / \mathrm{jmr} .2017 .18$ which crystallizes in the tungsten bronze structure, was reported to have an upper bound of zT that was on par with the layered cobaltites. ${ }^{6}$ The term "unfilled" refers to a tungsten bronze system in which only 5 out of the 6 A-sites of the structure are occupied. ${ }^{7}$

The high figure of merit of SBN was attributed to the remarkable increase in the electrical conductivity when processed under sufficiently-reducing atmospheres. ${ }^{8}$ While oxygen vacancies act as a dopant species contributing to the electrical conductivity of SBN, only modest increase were shown for annealing atmospheres of $10^{-6}$ through $10^{-12}$ atm $\mathrm{pO}_{2}$; the large increase in electrical conductivity was observed only after reaching an annealing condition of $10^{-14}$ atm $\mathrm{pO}_{2} .^{9}$ Coinciding with this increase in electrical conductivity was the precipitation of a $\mathrm{NbO}_{2}$ phase. ${ }^{9}$ Because the electrical conductivity of $\mathrm{NbO}_{2}$ is low between 300 and $600 \mathrm{~K}$, it should not be directly responsible for the large enhancement in conductivity. ${ }^{10}$ The large increase in conductivity was associated with the filling of the A-site vacancy in the structure, and the resulting generation of carriers. ${ }^{9,11,12}$ Here, the filling of the A-site acts as the other source of dopants. With respect to unfilled SBN, the effect of the A-site occupancy can be demonstrated with the expression:

$$
\begin{aligned}
5(\mathrm{Sr}, \mathrm{Ba})_{\mathrm{A}}{ }^{\times} & +\mathrm{V}_{\mathrm{A}}^{\times}+10 \mathrm{Nb}_{\mathrm{Nb}}^{\times}+(30-\delta) \mathrm{O}_{\mathrm{O}}^{\times} \leftrightarrow 5(\mathrm{Sr}, \mathrm{Ba})_{\mathrm{A}}^{\times} \\
& +(\mathrm{Sr}, \mathrm{Ba})_{\mathrm{V}_{\mathrm{A}}}+2 \mathrm{e}^{-}+10 \mathrm{Nb}_{\mathrm{Nb}}^{\times}+(30-\delta) \mathrm{O}_{\mathrm{O}}^{\times},
\end{aligned}
$$

where the 6 A-sites are denoted by the subscript, $A$ and the $\mathrm{V}_{\mathrm{A}}^{\times}$refers to the empty A-site in the unfilled SBN. ${ }^{12}$ The filling of the A-sites was supported by the 
observation that $\mathrm{NbO}_{2}$ precipitation was reversible, showing that neither $\mathrm{Ba}$ nor $\mathrm{Sr}$ were volatilizing out of the system. ${ }^{11}$ For unfilled SBNs, the amount of $\mathrm{NbO}_{2}$ precipitated (which is a function of $\mathrm{pO}_{2}$ and temperature) controlled the degree of A-site occupancy. ${ }^{11}$ It was found that $100 \%$ A-site occupancy could not be reached in unfilled SBN. However, Chan et al. and Kolodiazhnyi et al. have shown that the "filled" chemistry can be produced where all A-sites are occupied and where $\mathrm{NbO}_{2}$ is absent. ${ }^{12,13}$ This "filled" $\mathrm{SBN}\left(\mathrm{Sr}_{x} \mathrm{Ba}_{6-x} \mathrm{Nb}_{10} \mathrm{O}_{30-\delta}\right)$ is referred to as a "filled" bronze. ${ }^{7}$

While there is extensive literature on the electrical properties of SBN, most studies involve the unfilled composition of SBN. There are few studies with respect to the filled counterpart. Currently, only the lowtemperature thermoelectric properties of filled SBN have been reported. ${ }^{14}$ The present work fills this gap, with emphasis on the high-temperature thermoelectric properties of filled SBN. Due to the large increase in the electrical conductivity when A-sites are occupied in SBN, the Seebeck coefficients would be expected to be adversely affected. The results of Kolodiazhnyi et al. and $\mathrm{Li}$ et al. show that filled and partially filled SBNs possess low thermal conductivity from temperatures of $10-1000 \mathrm{~K}^{13,15}$ The effect on the power factor is thus a larger concern than the calculation of $\mathrm{zT}$ in this work.

\section{EXPERIMENTAL PROCEDURE}

\section{A. Ceramic processing}

The fabrication of dense ( $>95 \%$ of theoretical density for the barium end member $\left(\mathrm{Ba}_{6} \mathrm{Nb}_{10} \mathrm{O}_{30-\delta}\right.$ or $\left.\mathrm{BNO}\right)$ up to $60: 40 \mathrm{Sr}: \mathrm{Ba}$; and $\sim 93 \%$ of theoretical density for 80:20 Sr:Ba) SBN ceramics are given in previous work, but are summarized briefly here. ${ }^{12}$ Ceramic green bodies were formed by the isostatic pressing of filled SBN powders. The filled SBN powders were produced from the solid-state reaction of well-mixed high purity powders: $99.9 \%$ wt $\% \mathrm{SrCO}_{3}$ (Sigma-Aldrich, St. Louis, Missouri), 99.9 wt\% $\mathrm{Nb}_{2} \mathrm{O}_{5}$ (Alfa Aesar, Haverhill, Massachusetts), and $99.9 \mathrm{wt} \% \mathrm{BaCO}_{3}$ (Alfa Aesar) at $1300{ }^{\circ} \mathrm{C}$ for $10 \mathrm{~h}$ under a reducing atmosphere of $2.5 \%$ $\mathrm{H}_{2}$ balanced with Ar. The green bodies were then sintered at $1350{ }^{\circ} \mathrm{C}$ for $5 \mathrm{~h}$ under the same reducing atmosphere.

The sintered ceramics featured an average grain size between 2 and $3 \mu \mathrm{m}$ across all compositions with a bimodal grain size distribution. ${ }^{12}$ Though the 80:20 composition possessed lower densities relative to the theoretical value, no obvious differences in porosity were observed. Given the similarity in microstructure between all compositions, the microstructure is not expected to have a significant effect on electrical properties. Sintered pellets were cut into rectangular prisms of appropriate sizes for each of the electrical conductivity, Seebeck coefficient, and Hall Effect measurements. For electrical conductivity and
Seebeck samples, the sample sizes were $8.50-9.75 \mathrm{~mm}$, $1.30-1.80 \mathrm{~mm}$, and $1.00-1.30 \mathrm{~mm}$ for length, width, and thickness, respectively. Hall samples were $4.00-5.00 \mathrm{~mm}$, $1.00-1.50 \mathrm{~mm}$, and $1.00-1.40 \mathrm{~mm}$, for length, width, and thickness, respectively. Samples were then annealed between 1300 and $1310{ }^{\circ} \mathrm{C}$ for $30 \mathrm{~h}$ under a $\mathrm{pO}_{2} \sim 10^{-16} \mathrm{~atm}$ using $2.5 \% \mathrm{H}_{2}$ balanced with $\mathrm{Ar}$ gas or $\sim 10^{-14}$ atm $\mathrm{pO}_{2}$ with the addition of water vapor.

\section{B. Electrical measurements: electrical conductivity and Seebeck coefficient}

Electrical conductivities and Seebeck coefficients were obtained on a commercial Linseis LSR-3 unit (Linseis Inc., Robbinsville, New Jersey). Measurements were made using the differential method, employing a 4-point configuration, in which each of the four electrical leads also act as a thermocouple. In this method, a rectangular sample is loaded between two Pt blocks that supply the current for electrical conductivity measurements. Two inner probes measure the Seebeck voltage, the potential drop, and the temperature, such that the temperature gradient is calculated directly at the point of measurement. The temperature gradient and the global temperature were controlled by an auxiliary heater and infrared lamps located along the chamber, respectively.

Seebeck coefficients were measured from $50{ }^{\circ} \mathrm{C}$ to $700{ }^{\circ} \mathrm{C}(\sim 323$ to $\sim 973 \mathrm{~K})$ in increments of $50{ }^{\circ} \mathrm{C}$ with a heating rate of $3{ }^{\circ} \mathrm{C} / \mathrm{min}$ under an atmosphere of $1 \% \mathrm{H}_{2}$ balanced with $\mathrm{He}$. It is noted that this atmosphere will not further reduce the samples because the filled SBN samples were equilibrated under forming gas conditions at a higher temperature of $1300{ }^{\circ} \mathrm{C}$; use of a He atmosphere would actually oxidize the samples. Seebeck voltages were measured at 11 temperature gradients from $0{ }^{\circ} \mathrm{C}$ to $50{ }^{\circ} \mathrm{C}$ (relative to the top and bottom $\mathrm{Pt}$ leads) in gradient increments of $5{ }^{\circ} \mathrm{C}$. The dwell time for each of these measurements was $1 \mathrm{~min}$. The slope given by the Seebeck voltages and temperature gradients was used to calculate the Seebeck coefficient for each particular global temperature. Power factors were calculated from the conductivity and Seebeck coefficients.

4-Point probe electrical conductivities were also obtained independently using a Keithley digital multimeter (Keithley Instruments, Cleveland, Ohio). The temperature was controlled using a tube furnace. The reducing atmosphere was set with a gas mixture of $2.5 \% \mathrm{H}_{2}$ balanced with $\mathrm{N}_{2}$ during each measurement. Samples were electroded with $100 \mathrm{~nm}$ of Pt using a Quorum Technologies sputter coater (East Sussex, United Kingdom) and masked with Kapton tape. Epo-Tek Ag epoxy (resin + hardener in 1:1 ratio) was used to attach $\mathrm{Ag}$ wires onto the electrodes. The Ag epoxy was cured at $120{ }^{\circ} \mathrm{C}$ for $15 \mathrm{~min}$. Samples were electrically connected by physically twisting the $\mathrm{Ag}$ wire onto the Pt leads of the measurement apparatus. A heating program of $2{ }^{\circ} \mathrm{C} / \mathrm{min}$ ramp up from room 
temperature to $700{ }^{\circ} \mathrm{C}$, hold for $1 \mathrm{~h}$ at $700{ }^{\circ} \mathrm{C}$, and then a ramp down at $2{ }^{\circ} \mathrm{C} / \mathrm{min}$ was used while measurements were taken every $30 \mathrm{~s}$. Results were in good agreement with those measured on the LSR-3.

\section{Carrier concentration (Hall measurement)}

Carrier concentrations were quantified using a Quantum Design PPMS system (Quantum Design Inc., San Diego, California) via the Hall effect. A Hall-bar geometry with electrodes laid out in a 1-2-2-1 configuration was utilized. ${ }^{16} \mathrm{Ga}-$ In electrodes were used as Ohmic contacts. ${ }^{17}$ A Ga-In alloy of approximately 50:50 vol\% composition was painted onto each sample. Ag wires and Ag epoxy were used to attach leads to the electrodes. Sample leads were soldered onto a "Hall Puck" (i.e., the sample stage). The puck was loaded into the temperature-controlled vacuum chamber and sealed at $\sim 5$ mTorr. The temperature was maintained at $300.0 \pm 0.1 \mathrm{~K}$ and a $5000 \mu \mathrm{A} \mathrm{AC}$ current was used. Samples underwent two measurements starting from a magnetic field of 80,000 Oe to $-80,000 \mathrm{Oe}$ and then from $-80,000$ Oe back to 80,000 Oe at a rate of $100 \mathrm{Oe} / \mathrm{s}$. Hall resistances $\left(R_{X Y}\right)$ were measured every 10,000 Oe. The error due to electrode misalignment was corrected by this reversal of the magnetic field, since this contribution was not dependent on the magnetic field.

The magnetic permeability for filled and unfilled SBN have not yet been reported in the literature. However, Sienko et al. and Kupka et al. reported the magnetic susceptibilities for many of the sodium, potassium, and rubidium tungsten bronzes, which are on the order of $10^{-6} \cdot{ }^{18,19}$ Thus, the relative magnetic permeability of the present samples was taken to be unity (it can be shown that for a relative permeability of unity, the induced magnetic field $\mathrm{B}$, can be equated to the auxiliary magnetic field $\mathrm{H}$ if the units of gauss $\left(10^{-4}\right.$ tesla) and oersted are used, respectively $\left.{ }^{20}\right)$.

The carrier concentration, $n$, was calculated from the slope of the Hall resistance and the applied magnetic field

$$
R_{X Y}=B_{Z} \times \frac{1}{n e d}
$$

where: $\mathrm{R}_{X Y}$ is the Hall resistance across the width of the sample that is derived from the current flowing in the $X$-direction $\left(I_{X}\right)$ and the Hall voltage measured in the $Y$-direction $\left(V_{Y}\right)$ where the directions, $X$ and $Y$, are parallel to the axis and width of the sample, respectively. $B_{Z}$ is the applied magnetic field in the Z-direction, which is parallel to the thickness direction. The symbol e denotes the elementary charge and $d$ is the thickness.

\section{RESULTS AND DISCUSSION}

The electrical conductivities of oxygen-deficient filled SBNs are shown in Fig. 1(a). The trend in electrical conductivity, $\sigma$, with temperature, $T$, is similar to that reported in previous works on highly-reduced unfilled SBN compositions. ${ }^{8,9,15,21}$ In the unfilled compositions, a $\frac{\mathrm{d} \sigma}{\mathrm{d} T}>0$ is observed generally between 200 and $650 \mathrm{~K}$ before reaching a maximum conductivity, which is then followed by $\frac{\mathrm{d} \sigma}{\mathrm{d} T}<0$ with increasing temperature. While this behavior is observed in Fig. 1(a), two major differences from the unfilled compositions are apparent. The maximum conductivities for filled SBNs (for a Sr:Ba ratio less than 80:20) are greater by a factor of $2-3$, and the room temperature conductivities are greater by one order of magnitude compared to similarly-reduced unfilled SBNs reported in literature. For example, in the work of Bock et al., the 60:40 Sr:Ba composition annealed at $1300{ }^{\circ} \mathrm{C}$, and $\sim 10^{-16}$ atm $\mathrm{pO}_{2}$ had room temperature and maximum electrical conductivities of about $30 \mathrm{~S} / \mathrm{cm}$ and $275 \mathrm{~S} / \mathrm{cm}$, respectively. In contrast, the filled 60:40 in Fig. 1(a) shows values of about $250 \mathrm{~S} / \mathrm{cm}$ and $575 \mathrm{~S} / \mathrm{cm}$, respectively. Similarly, Li et al. reported the room temperature and maximum conductivity of the unfilled 40:60 composition annealed at $1300{ }^{\circ} \mathrm{C}$ and $\sim 10^{-16}$
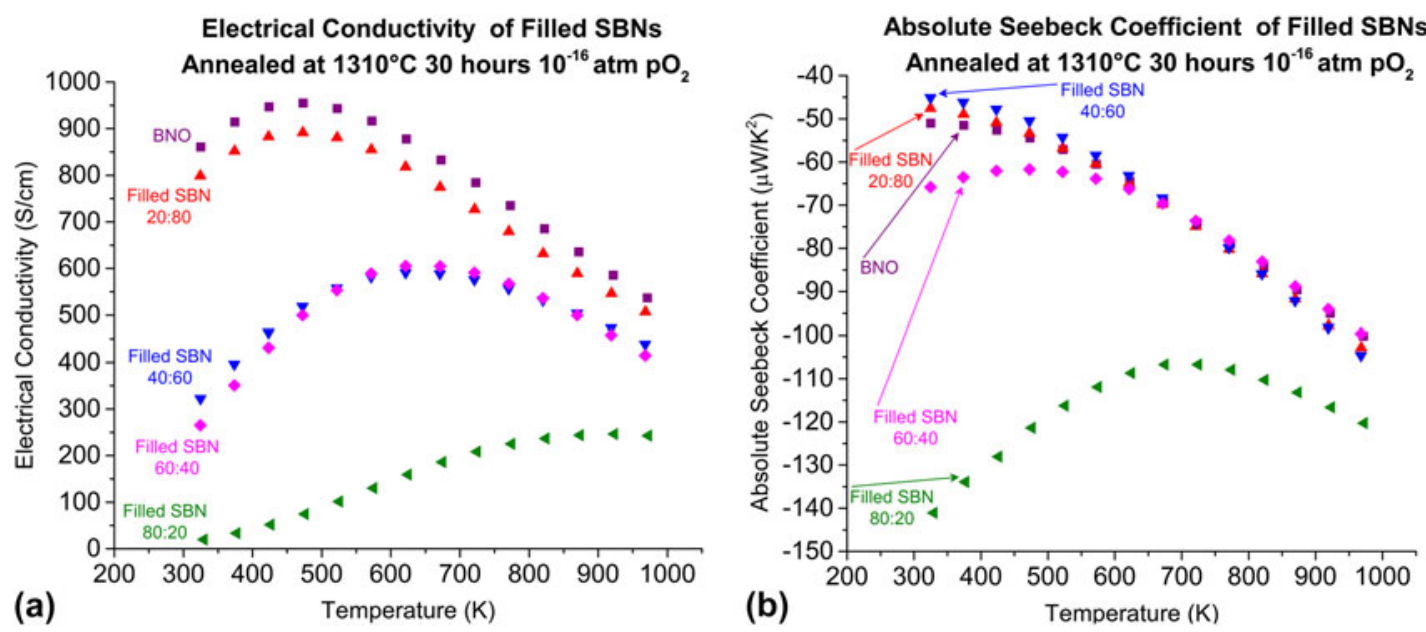

FIG. 1. Electrical conductivity (a) and absolute Seebeck coefficients (b) of filled oxygen-deficient SBNs. 
atm $\mathrm{pO}_{2}$ to be $33 \mathrm{~S} / \mathrm{cm}$ and $120 \mathrm{~S} / \mathrm{cm}$, respectively. The filled 40:60 composition, as shown in Fig. 1(a), demonstrates room temperature and maximum conductivities of about $300 \mathrm{~S} / \mathrm{cm}$ and $500 \mathrm{~S} / \mathrm{cm}$, respectively.

Despite the difference in magnitude of the conductivities between the filled and unfilled SBNs, the shape of the curves in Fig. 1(a) indicates that the conduction mechanism in the filled SBNs is similar to their unfilled counterparts. A band structure describing conduction in oxygen-deficient unfilled SBN was proposed by Bock et al. ${ }^{9}$ As a consequence of high disorder in these materials, Anderson localization modifies the band structure via the presence of mobility edges. The temperature dependence of the Seebeck coefficients, as shown in Fig. 1(b), also supports this hypothesis.

The Heike formula for hopping conductors models a temperature-independent Seebeck coefficient, which does not fit the experimental data for the filled bronzes over the temperature range shown in Fig. 1(b). The electrical conductivities are in the hundreds of $\mathrm{S} / \mathrm{cm}$, which is higher than typical hopping conductors. ${ }^{22}$ In addition, the carrier mobility, as calculated from the Hall measurement for filled 60:40 SBNs, is above the $0.1 \mathrm{~cm}^{2} / \mathrm{V} \mathrm{s}$ threshold for typical hopping conductors. Therefore, hopping conduction can be ruled out within the measured temperature range. ${ }^{23}$ The Seebeck coefficients shown in Fig. 1(b) are consistent with the measured electrical conductivities, in that higher absolute values of the Seebeck coefficient are observed in compositions with lower conductivities. The parabolic-like behavior seen in the Sr-rich compositions is also observed in the unfilled SBNs and was attributed to relaxor ferroelectricity. ${ }^{9}$ The idea that relaxor ferroelectricity is also causing this parabolic behavior in the filled SBNs has not yet been verified. Hessen et al. and Kolodiazhnyi et al. reported that BNO and the filled SBNs fit into the centrosymmetric $P 4 / \mathrm{mbm}$ space group through X-ray diffraction, though other techniques to test for centrosymmetry have not yet been conducted for these materials.

As the $\mathrm{Sr}$ content is decreased, a left-upward shift can be seen for the electrical conductivities in Fig. 1(a). This is observed in the findings of Bock et al., and Dandeneau et al., in the unfilled counterparts, where the temperature of the maximum conductivity shifted to lower temperatures for more-reduced samples (i.e., larger carrier densities) and for lower $\mathrm{Sr}$ contents. ${ }^{9,21}$ Compositions with higher $\mathrm{Sr}$ were reported to have lower conductivities at lower temperatures and/or showed lower maxima in the conductivity, while greater degrees of reduction led to higher values in maximum conductivity. ${ }^{9,15,21}$ Bock et al. also showed that the activation energies for conduction increase as the $\mathrm{Sr}$ content increases, consistent with the observed left-upward shift. ${ }^{9}$ The higher activation energies were attributed to the effect of relaxor ferroelectricity on electron transport. If the oxygen-deficient filled SBNs are noncentrosymmetric (at least locally), then it is possible that one cause for the left-upward shifts in electrical conductivity shown in Fig. 1(a) is due to relaxor behavior.

The maximum electrical conductivity also increases with decreasing $\mathrm{Sr}$ content and invites speculation on how the mobility and the carrier concentration are affected by composition. With regard to the former, Lee et al. and Hessen et al. showed that the $c$-axis is the high conductivity axis. ${ }^{8,24}$ The anisotropy is consistent with the Goodenough model, since $\mathrm{Nb}-\mathrm{O}-\mathrm{Nb}$ bond angles critical for orbital overlap along this direction are closest to $180^{\circ} .{ }^{25}$ Octahedral tilts in SBN would result in a deviation from the ideal $180^{\circ}$. If the bond angles along the $c$-axis affect the electrical conductivity, it is assumed to be a result of increased carrier mobility, since octahedral tilting should not generate additional carriers.

Both Jamieson and Trubelja et al. conjecture that the degree of octahedral tilting increases with $\mathrm{Sr}$ content in SBN, with larger $\mathrm{Ba}$ atoms producing a more "up-right structure". 26,27 The observation of compositions with higher $\mathrm{Sr}$ exhibiting lower conductivities is consistent with this notion. Figure 2 shows electron diffraction patterns of the filled barium end member and the filled 60:40 compositions taken on a Philips EM420 microscope operated at $120 \mathrm{kV}$ (FEI Company, Hillsboro, Oregon). The filled 60:40 composition exhibits commensurate superlattice diffraction along the $c$-axis and an incommensuration along [110], which has been observed in other tungsten bronzes. ${ }^{28,29}$ Superlattice diffraction spots were not observed in BNO, implying the absence of octahedral tilting in the barium end member. This suggests that there may be a correlation between octahedral tilting and carrier mobility.

At the same time, it is possible that for a given reduction condition, different compositions (i.e., Sr:Ba ratios and filled versus unfilled) could possess a different carrier density. To determine this, Hall measurements were conducted to quantify the carrier concentration as a function of $\mathrm{pO}_{2}$. Each set of raw data showed a negative slope in Hall resistance versus magnetic field, reflecting a negative Hall coefficient. This agrees with the negative Seebeck coefficients in Fig. 1(b) and the notion that oxygen-deficient SBN is an n-type conductor. The results of the carrier density for filled and unfilled 60:40 SBN ceramics are shown in Fig. 3. At $10^{-16}$ atm $\mathrm{pO}_{2}$, the carrier concentrations of SBN 60:40 are $n_{\text {Filled }}=2.32 \times 10^{21} \pm 3.4 \times 10^{20}$ carriers per $\mathrm{cm}^{3}$ and $n_{\text {Unfilled }}=2.20 \times 10^{21} \pm 4.2 \times 10^{20}$ carriers per $\mathrm{cm}^{3}$. The quantitative energy dispersive spectroscopy results from Bock et al. showed that the conductivity increased as a function of the amount of $\mathrm{NbO}_{2}$ precipitated, and hence the extent of A-site filling. ${ }^{11}$ Annealing at $1300{ }^{\circ} \mathrm{C}$ and $10^{-16}$ atm $\mathrm{pO}_{2}$ does not precipitate the full amount of $\mathrm{NbO}_{2}$, leaving $\sim 80 \%$ of the final A-sites unfilled. If the model of electronic compensation for A-site filling is 


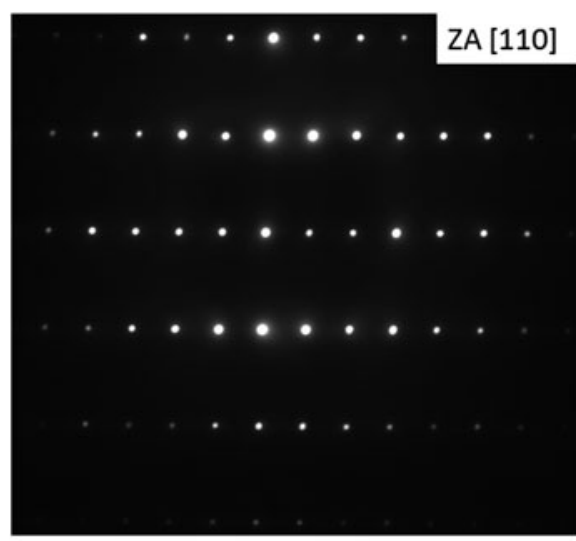

BNO 110 Zone-Axis

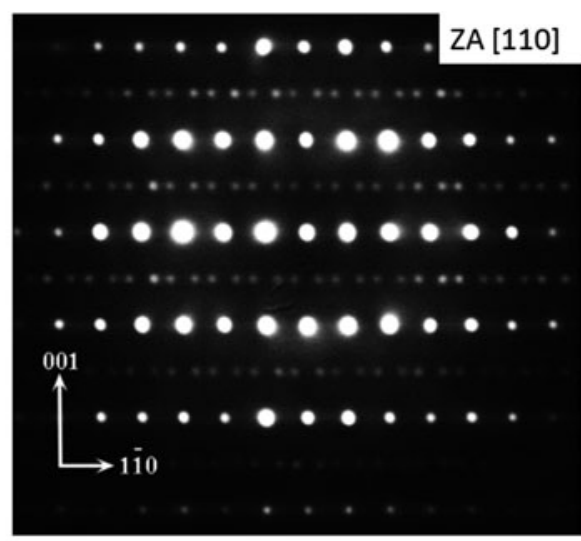

Filled SBN 60:40 110 Zone-Axis

FIG. 2. Electron diffraction patterns of filled SBN viewed along [110] zone-axis. BNO exhibits no superlattice diffraction spots, while the 60:40 composition shows the presence of superlattice diffraction spots. Both diffraction patterns were taken at a camera length of $320 \mathrm{~mm}$ and an accelerating voltage of $120 \mathrm{kV}$.

\section{Carrier Concentration vs Annealing Conditions at $1300^{\circ} \mathrm{C}$}

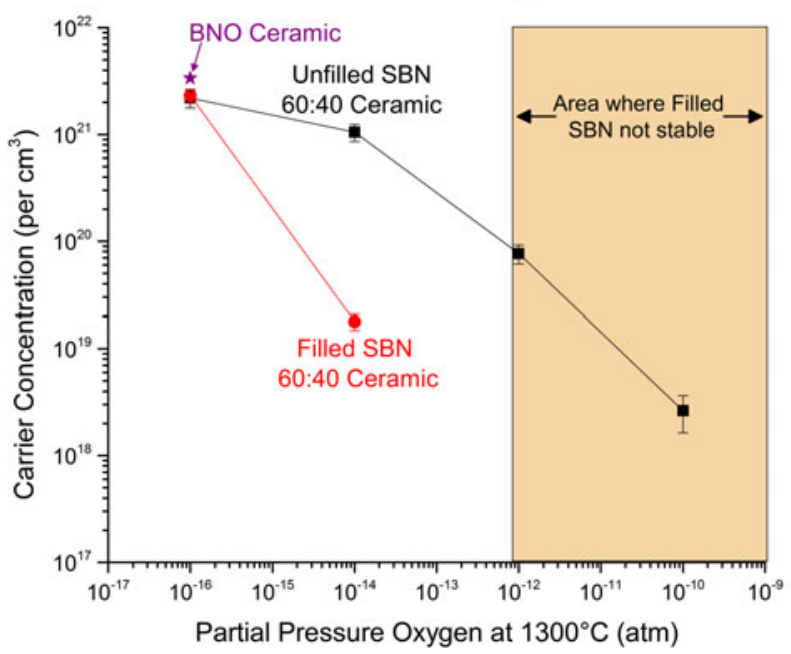

FIG. 3. Carrier concentration versus annealing condition at $1300{ }^{\circ} \mathrm{C}$ between filled and unfilled 60:40 SBN ceramics. Note the double log scale. Result for BNO is indicated by a star-shaped symbol.

correct, then there is a difference of $\sim 6.3 \times 10^{20}$ carriers per $\mathrm{cm}^{3}$ between an unfilled and filled 60:40 SBN annealed $10^{-16}$ atm $\mathrm{pO}_{2}$ conditions. This difference in carriers is within error of the Hall measurement. However, the carrier concentration alone does not explain the stark difference in maximum conductivity $(\sim 600-650 \mathrm{~K})$ between the filled and unfilled SBNs, which differ by a factor of 5 and 2 at room temperature and at the maxima, respectively (Fig. 4).

Though the presence of $\mathrm{NbO}_{2}$ as a secondary phase may indirectly limit the carriers generated from A-site filling, it is speculated that it may also lower the conductivity by lessening the contribution of the high conductivity $c$-axis. Reduction of unfilled SBN single crystals showed that $\mathrm{NbO}_{2}$ preferentially forms on the surface perpendicular to the $c$-axis. ${ }^{30}$ The electrical conductivity of $\mathrm{NbO}_{2}$ was reported to be $40 \mathrm{~S} / \mathrm{cm}$ at about $650 \mathrm{~K} .{ }^{10}$ Since SBN has a higher conductivity along the $c$-axis, the nucleation of more resistive $\mathrm{NbO}_{2}$ at the $c$-axis surface could potentially affect the conduction across grain boundaries in ceramic samples. The conductivity of $\mathrm{NbO}_{2}$ as reported by Janninck et al. increases drastically at higher temperatures to $\sim 250 \mathrm{~S} / \mathrm{cm}$ at 1000 $\mathrm{K}$ and $\sim 500 \mathrm{~S} / \mathrm{cm}$ at $\sim 1300 \mathrm{~K} .{ }^{10}$ The abrupt increase in conductivity in $\mathrm{NbO}_{2}$ may also explain why the conductivity of unfilled SBN at higher temperatures falls off less slowly than the filled SBN as shown in Fig. 4.

Unlike the unfilled compositions, where hopping conduction was observed between 200 and $290 \mathrm{~K}$, the filled compositions have almost half the carriers activated above the mobility edge. ${ }^{9}$ The result is that band conduction dominates even near room temperatures. This is demonstrated by the high conductivities near room temperature (Fig. 4). Given the similar order of magnitude for the carrier densities, the reason for the lower activation energy for filled relative to the unfilled SBNs, is currently unknown. The maximum in electrical conductivity corresponds to the saturation point, in which all carriers from the impurity band have been excited over the mobility edge and into the $\mathrm{Nb} 4 d$ conduction band. Additional increases in temperature lower the conductivity as a result of a net decrease in charge carrier mobility due to scattering in the absence of carrier excitation.

At $10^{-14}$ atm $\mathrm{pO}_{2}$, the carrier concentration differs by almost two orders of magnitude between the filled and unfilled SBN. The lower carrier concentration is consistent with Fig. 4, in which the electrical conductivities of the filled 60:40 were lower by two orders of magnitude at room temperature and an order of magnitude at $600 \mathrm{~K}$ relative to the $10^{-16}$ atm $\mathrm{pO}_{2}$ counterparts. Furthermore, the filled SBN does not reach a maximum in electrical conductivity in this temperature range. The reason for the marked difference between filled and unfilled SBN at 


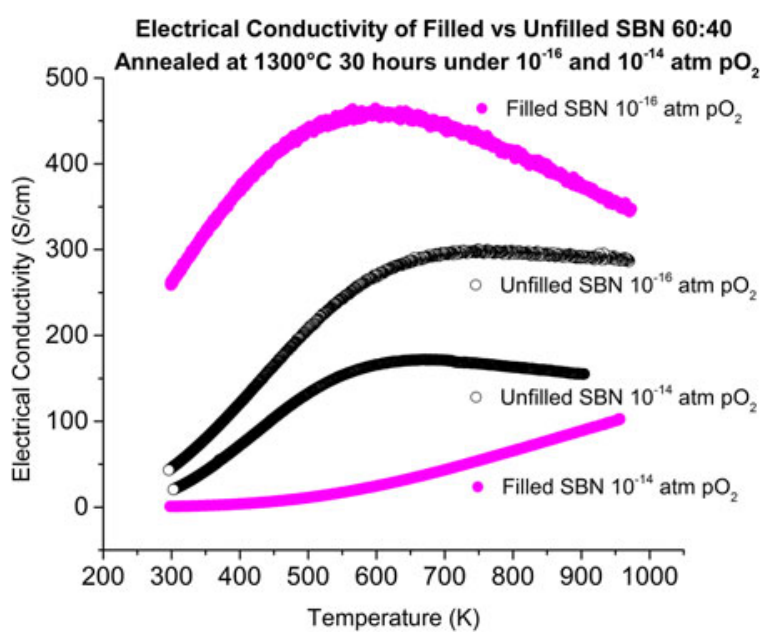

FIG. 4. Electrical conductivity: comparison between filled and unfilled 60:40 SBN annealed at $1300{ }^{\circ} \mathrm{C}$ for $30 \mathrm{~h}$ under $10^{-16}$ atm $\mathrm{pO}_{2}$ and $10^{-14}$ atm $\mathrm{pO}_{2}$.

$10^{-14}$ atm $\mathrm{pO}_{2}$ is not yet known, but two plausible cases to be considered are that at higher $\mathrm{pO}_{2}$ of $10^{-14} \mathrm{~atm}$, filled SBN grain boundaries are more easily oxidized without the presence of $\mathrm{NbO}_{2}$, resulting in an increase in the grain boundary resistance. Another possibility is the ionic compensation of A-site filling through superoxidation, as suggested by Bock et al. ${ }^{11}$

Though it has been surmised that the high conductivity of the BNO ceramic shown in Fig. 1(a) could be due to the difference in carrier mobility, Fig. 3 also indicates that a higher carrier concentration is seen in BNO. The carrier concentration was found to be greater than the filled 60:40 SBN by a factor of 1.45 , where $n_{\mathrm{BNO}-16}=3.37 \times 10^{21} \pm 1.0 \times 10^{20}$ carriers per $\mathrm{cm}^{3}$. Thus, an increase in both carrier mobility and carrier density may be responsible for Ba-rich compositions possessing higher conductivities for the given reduction condition.

The carrier concentration data in Fig. 3 suggests that SBN does not follow an optimal carrier concentration between $1.0 \times 10^{19}$ and $10^{20}$ carriers per $\mathrm{cm}^{3}$, as is typical of most thermoelectrics. ${ }^{31}$ In much the same way, the sodium cobaltites have displayed carrier densities on the order of $\sim 10^{22}$ carriers per $\mathrm{cm}^{3}{ }^{32}$ It is inferred from Figs. 3 and 4 that SBN with $10^{21}$ carriers per $\mathrm{cm}^{3}$ would exhibit better thermoelectric performance than with $10^{19}$ carriers per $\mathrm{cm}^{3}$.

The power factors calculated from Figs. 1(a) and 1(c) are shown in Fig. 5. The power factor as a function of temperature in the filled SBNs follows a similar trend and magnitude to the unfilled SBNs reported by $\mathrm{Li}$ et al.: power factors of around $3.0 \mu \mathrm{W} /\left(\mathrm{cm} \mathrm{K}^{2}\right)$ at $700 \mathrm{~K}$ for both the filled and unfilled 60:40 composition. ${ }^{15}$ However, at higher temperatures, the lower Sr compositions of the filled SBNS are higher; at $1000 \mathrm{~K}, 4.6 \mu \mathrm{W} /\left(\mathrm{cm} \mathrm{K}^{2}\right)$,

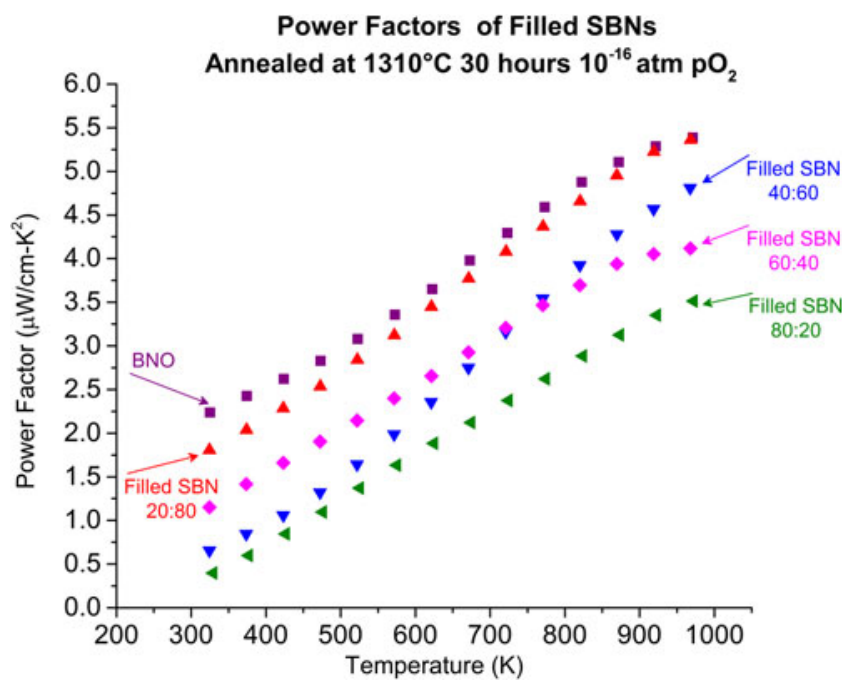

FIG. 5. Power factors of filled SBNs calculated from Figs. 1(a) and 1(b).

compared to $3.45 \mu \mathrm{W} /\left(\mathrm{cm} \mathrm{K}^{2}\right)$ for filled and unfilled 40:60 composition, respectively. ${ }^{15}$ Despite the large carrier densities, the BNO and 20:80 filled SBNs show higher power factors than any unfilled SBNs or La-doped unfilled SBNs reported in the literature, at temperatures from 300 to $1000 \mathrm{~K}^{15,33}$ The current trend shows that power factors continue to increase beyond the measurement range shown here, suggesting that filled SBN may be useful as a high-temperature thermoelectric, provided they are used in low oxygen environments or if they can be effectively protected by a coating.

\section{SUMMARY AND CONCLUSIONS}

The thermoelectric properties of heavily-reduced filled SBNs were evaluated from $\sim 350$ to $970 \mathrm{~K}$. The conduction mechanism of the filled SBN compositions was found to be band-conduction at higher temperatures. However, filled SBNs, particularly those of Ba-rich compositions, showed higher electrical conductivities than their unfilled counterparts. This is believed to be due to increases in carrier density and carrier mobility. Higher conductivities near room temperature observed in low $\mathrm{Sr}$ compositions may be attributed to the onset of relaxor ferroelectricity as the $\mathrm{Sr}$ content is increased. The high electrical conductivities shown by the filled SBNs of low $\mathrm{Sr}$ composition have produced the highest reported power factors for SBN ceramics. If the thermal conductivities of the partially filled SBNs measured by Li. et al. are used to calculate zT, then the zT for the filled 60:40 composition at $\sim 970 \mathrm{~K}$ is about 0.21 . In the context of other well-performing polycrystalline oxide thermoelectrics, the zTs of $\mathrm{Na}_{x} \mathrm{CoO}_{2}, \mathrm{Sr}_{1-x} \mathrm{La}_{x} \mathrm{TiO}_{3}$, and $\mathrm{Zn}_{0.98} \mathrm{Al}_{0.02} \mathrm{O}$ are reported to be: $0.80(950 \mathrm{~K}), 0.20-0.37(600-750 \mathrm{~K})$, and $0.65(1073 \mathrm{~K})$, respectively. ${ }^{34-37}$ 


\section{ACKNOWLEDGMENTS}

This research was funded by the National Science Foundation under grant number DMR-1206518. The author would also like to thank Wei Wei Zhao of the Moses Chan group from The Pennsylvania State University for assistance in the Hall measurements.

\section{REFERENCES}

1. S. LeBlanc, S.K. Yee, M.L. Scullin, C. Dames, and K.E. Goodson: Material and manufacturing cost considerations for thermoelectrics. Renewable Sustainable Energy Rev. 32, 313 (2014).

2. S.B. Riffat and X. Ma: Thermoelectrics: A review of present and potential applications. Appl. Therm. Eng. 23(8), 913 (2003).

3. R.U. Ayres and L. Ayres: A Handbook of Industrial Ecology (Edward Elgar Publishing, Northhampton, MA, 2002).

4. S.K. Bux, R.G. Blair, P.K. Gogna, H. Lee, G. Chen, M.S. Dresselhaus, R.B. Kaner, and J. Fleurial: Nanostructured bulk silicon as an effective thermoelectric material. Adv. Funct. Mater. 19(15), 2445 (2009).

5. S. Walia, S. Balendhran, H. Nili, S. Zhuiykov, G. Rosengarten, Q.H. Wang, M. Bhaskaran, S. Sriram, M.S. Strano, and K. Kalantar-zadeh: Transition metal oxides-Thermoelectric properties. Prog. Mater. Sci. 58(8), 1443 (2013).

6. S. Lee, J.A. Bock, S. Trolier-McKinstry, and C.A. Randall: Ferroelectric-thermoelectricity and Mott transition of ferroelectric oxides with high electronic conductivity. J. Eur. Ceram. Soc 32(16), 3971 (2012)

7. X. Zhu, M. Fu, M.C. Stennett, P.M. Vilarinho, I. Levin, C.A. Randall, J. Gardner, F.D. Morrison, and I.M. Reaney: A crystal-chemical framework for relaxor versus normal ferroelectric behavior in tetragonal tungsten bronzes. Chem. Mater. 27(9), 3250 (2015).

8. S. Lee, R.H.T. Wilke, S. Trolier-McKinstry, S. Zhang, and C.A. Randall: $\mathrm{Sr}_{x} \mathrm{Ba}_{1-x} \mathrm{Nb}_{2} \mathrm{O}_{6-\delta}$ ferroelectric-thermoelectrics: Crystal anisotropy, conduction mechanism, and power factor. Appl. Phys. Lett. 96(3), 31910 (2010).

9. J.A. Bock, S. Trolier-McKinstry, G.D. Mahan, and C.A. Randall: Polarization-based perturbations to thermopower and electronic conductivity in highly conductive tungsten bronze structured ( $\mathrm{Sr}, \mathrm{Ba}) \mathrm{Nb}_{2} \mathrm{O}_{6}$ : Relaxors vs normal ferroelectrics. Phys. Rev. B: Condens. Matter Mater. Phys. 90(11), 115106 (2014).

10. R.F. Janninck and D.H. Whitmore: Electrical conductivity and thermoelectric power of niobium dioxide. J. Phys. Chem. Solids 27(6-7), 1183 (1966).

11. J.A. Bock, J.H. Chan, Y. Tsur, S. Trolier-McKinstry, and C.A. Randall: The effects of low oxygen activity conditions on the phase equilibria and cation occupancy of strontium barium niobate. J. Am. Ceram. Soc. 99(10), 3435 (2016).

12. J.H. Chan, J.A. Bock, H. Guo, S. Trolier-McKinstry, and C.A. Randall: Filled oxygen-deficient strontium barium niobates. J. Am. Ceram. Soc. (2016), doi: 10.1111/jace.14598.

13. T. Kolodiazhnyi, H. Sakurai, O. Vasylkiv, H. Borodianska, and Y. Mozharivskyj: Abnormal thermal conductivity in tetragonal tungsten bronze $\mathrm{Ba}_{6-x} \mathrm{Sr}_{x} \mathrm{Nb}_{10} \mathrm{O}_{30}$. Appl. Phys. Lett. 104(11), 111903 (2014).

14. T. Kolodiazhnyi, H. Sakurai, M. Isobe, Y. Matsushita, S. Forbes, Y. Mozharivskyj, T.J.S. Munsie, G.M. Luke, M. Gurak, and D.R. Clarke: Superconductivity and crystal structural origins of the metal-insulator transition in $\mathrm{Ba}_{6-x} \mathrm{Sr}_{x} \mathrm{Nb}_{10} \mathrm{O}_{30}$ tetragonal tungsten bronzes. Phys. Rev. B: Condens. Matter Mater. Phys. 92(21), 214508 (2015)
15. Y. Li, J. Liu, Y. Hou, Y. Zhang, Y. Zhou, W. Su, Y. Zhu, J. Li, and C. Wang: Thermal conductivity and thermoelectric performance of $\mathrm{Sr}_{x} \mathrm{Ba}_{1-x} \mathrm{Nb}_{2} \mathrm{O}_{6}$ ceramics at high temperatures. Scr. Mater. 109, 80 (2015).

16. S. Jandl, K.D. Usadel, and G. Fischer: Resistivity measurements with samples in the form of a double cross. Rev. Sci. Instrum. 45(11), 1479 (1974).

17. R.J. Cava, W.F. Peck, Jr, J.J. Krajewski, and D.A. Fleming: Compensation of the temperature coefficient of the dielectric constant of barium strontium titanate. Appl. Phys. Lett. 67(25), 3813 (1995)

18. F. Kupka and M.J. Sienko: The magnetic susceptibility of tungsten bronzes. J. Chem. Phys. 18(9), 1296 (1950).

19. M.J. Sienko and S. Macenness Morehouse: Electrical and magnetic properties of potassium tungsten bronze and rubidium tungsten bronze. Inorg. Chem. 2(3), 485 (1963).

20. W.D. Callister and D.G. Rethwisch: Materials Science and Engineering: An Introduction, 8th ed. (John Wiley \& Sons, Hoboken, 2009).

21. C.S. Dandeneau, T.W. Bodick, R.K. Bordia, and F.S. Ohuchi: Thermoelectric properties of reduced polycrystalline $\mathrm{Sr}_{0.5} \mathrm{Ba}_{0.5} \mathrm{Nb}_{2} \mathrm{O}_{6}$ fabricated via solution combustion synthesis. J. Am. Ceram. Soc. 96(7), 2230 (2013).

22. M. Cutler and N.F. Mott: Observation of Anderson localization in an electron gas. Phys. Rev. 181(3), 1336 (1969).

23. A.J. Bosman and H.J. Van Daal: Small-polaron versus band conduction in some transition-metal oxides. Adv. Phys. 19(77), 1 (1970).

24. B. Hessen, S.A. Sunshine, T. Siegrist, A.T. Fiory, J.V. Waszczak: Structure and properties of reduced barium niobium oxide single crystals obtained from borate fluxes. Chem. Mater. 3(16), 528 (1991).

25. O.G. D'yachenko, S.Y. Istomin, A.M. Abakumov, and E.V. Antipov: Synthesis, structure, and properties of mixed niobium(IV,V) oxides. Inorg. Mater. 36(3), 247 (2000).

26. P.B. Jamieson: Ferroelectric tungsten bronze-type crystal structures. I. Barium strontium niobate $\mathrm{Ba}_{0.27} \mathrm{Sr}_{0.75} \mathrm{Nb}_{2} \mathrm{O}_{5.78}$. J. Chem. Phys. 48(11), 5048 (1968).

27. M. Trubelja, E. Ryba, and D.K. Smith: A study of positional disorder in strontium barium niobate. J. Mater. Sci. 31, 1435 (1996).

28. I. Levin, M.C. Stennett, G.C. Miles, D.I. Woodward, A.R. West, and I.M. Reaney: Coupling between octahedral tilting and ferroelectric order in tetragonal tungsten bronze-structured dielectrics. Appl. Phys. Lett. 89(12), 122908 (2006).

29. C.A. Randall, R. Guo, A.S. Bhalla, and L.E. Cross: Microstructure-property relations in tungsten bronze lead barium niobate, $\mathrm{Pb}_{1-x} \mathrm{Ba}_{x} \mathrm{Nb}_{2} \mathrm{O}_{6}$. J. Mater. Res. 6(8), 1720 (1991).

30. J.A. Bock: Investigation of Reduced $\left(\mathrm{Sr}_{x} \mathrm{Ba}_{1-x}\right) \mathrm{Nb}_{2} \mathrm{O}_{6}$ as a Ferroelectric-Based Thermoelectric. Ph.D. Thesis, Pennsylvania State University, 2016.

31. G.J. Snyder and E.S. Toberer: Complex thermoelectric materials. Nat. Mater. 7(2), 105 (2008).

32. I. Terasaki: Layered cobalt oxides: correlated electrons for thermoelectrics. In Thermoelectr. Nanomater. SE - 3, K. Koumoto and T. Mori, eds. (Springer, Berlin, 2013); pp. 51-70.

33. Y. Li, J. Liu, Y. Zhang, Y. Zhou, J. Li, W. Su, J. Zhai, H. Wang, and $\mathrm{C}$. Wang: Enhancement of thermoelectric performance of $\mathrm{Sr}_{0.7} \mathrm{Ba}_{0.3} \mathrm{Nb}_{2} \mathrm{O}_{6-\delta}$ ceramics by lanthanum doping. Ceram. Int. 42(1), 1128 (2016).

34. M. Ito, T. Nagira, D. Furumoto, S. Katsuyama, and H. Nagai: Synthesis of $\mathrm{Na}_{x} \mathrm{Co}_{2} \mathrm{O}_{4}$ thermoelectric oxides by the polymerized complex method. Scr. Mater. 48(4), 403 (2003). 
35. S. Ohta, T. Nomura, H. Ohta, and K. Koumoto: Hightemperature carrier transport and thermoelectric properties of heavily La-or Nb-doped $\mathrm{SrTiO}_{3}$ single crystals. J. Appl. Phys. 97(3), 34106 (2005).

36. S. Funahashi, T. Nakamura, K. Kageyama, and H. Ieki: Monolithic oxide-metal composite thermoelectric generators for energy harvesting. J. Appl. Phys. 109(12), 124509 (2011).

37. M. Ohtaki and R. Hayashi: Enhanced Thermoelectric Performance of Nanostructured $\mathrm{ZnO}$ : A possibility of selective phonon scattering and carrier energy filtering by nanovoid structure. In Thermoelectr. 2006. ICT'06. 25th Int. Conf. (IEEE, Vienna, 2006), pp. 276-279. 